\title{
Heterotopic Ossification after Knee Surgery, Arthroscopy is Not an Exception!!!!
}

\author{
S N Lokesh Kumar ${ }^{1}$, Santosh Ravi ${ }^{1}$, K N Subramaniam², S N Jagadesh Kumar ${ }^{3}$, Amavarapu \\ Sowmya Priya ${ }^{4}$
}

\section{Learning Point of the Article:}

Arthroscopic surgeries are not $\mathrm{n}$ exception for heterotopic ossification. Clinical suspicion and meticulous physical examination is needed to avoid such complications.

\section{Abstract}

Introduction: Reconstruction of the anterior cruciate ligament (ACL) is a common procedure. Injury is the predisposing risk factor for developing heterotopic ossification $(\mathrm{HO})$. We like to report a case of $\mathrm{HO}$ of the knee following arthroscopic ACL reconstruction.

Case Report: A29-year-old patient was admitted with complaints of painful instability of the left knee after a fall from bike. MRI study confirmed a complete ACL rupture along with tear of the body and posterior horns of the medial meniscus. ACL reconstruction was carried out using ipsilateral hamstring tendon graft by knee arthroscopy. Eight months after the procedure, the patient complained of pain in the lateral side of the knee joint evidencing a slight prominence of hard consistency. The radiological study confirmed it to be a case of $\mathrm{HO}$. The patient was managed conservatively.

Conclusion: Bone is the only tissue that has an incredible property as such that it can differentiate and develop into its mature form outside its native locations. Numerous studies have suggested ways of preventing and treating this complication. One has to be aware that even these simple surgical procedures can lead on to $\mathrm{HO}$.

Keywords: Heterotopic ossification in arthroscopy, unusual complication in arthroscopy, heterotopic ossification, knee arthroscopy complication.

\section{Introduction}

Reconstruction of the anterior cruciate ligament (ACL) is one of the most common procedures carried out by arthroscopy surgeons. Although the complications after ACLare low, it is not exempt. Infection, stiffness, nerve paresthesia, and residual instability are well-known complications. We like to report a case of heterotopic ossification $(\mathrm{HO})$ of the knee following arthroscopic ACL reconstruction using a quadrupled hamstring graft. Although $\mathrm{HO}$ has been well reported following surgery of the acetabulum or hip, HO following arthroscopic knee surgery has not been reported frequently.

\section{Case Report}

A 29-year-old male complained of pain, swelling, and instability of the left knee starting 6 months ago, after a fall while riding a motorbike. The physical examination exhibited a positive Lachman test, anterior drawer test, positive pivot shift test along with medial joint line tenderness, and a positive Mc Murray test, indicating an ACL tear with meniscal injury. He had full range of movement. There were normal radiological findingsin the knee $\mathrm{X}$-ray. The MRI study confirmed a complete ACL rupture along with tear of the body and posterior horns of the medial meniscus and a partial tear of the posterior cruciate ligament (PCL). After obtaining informed consent, ACL reconstruction was carried out using ipsilateral hamstring tendon graft

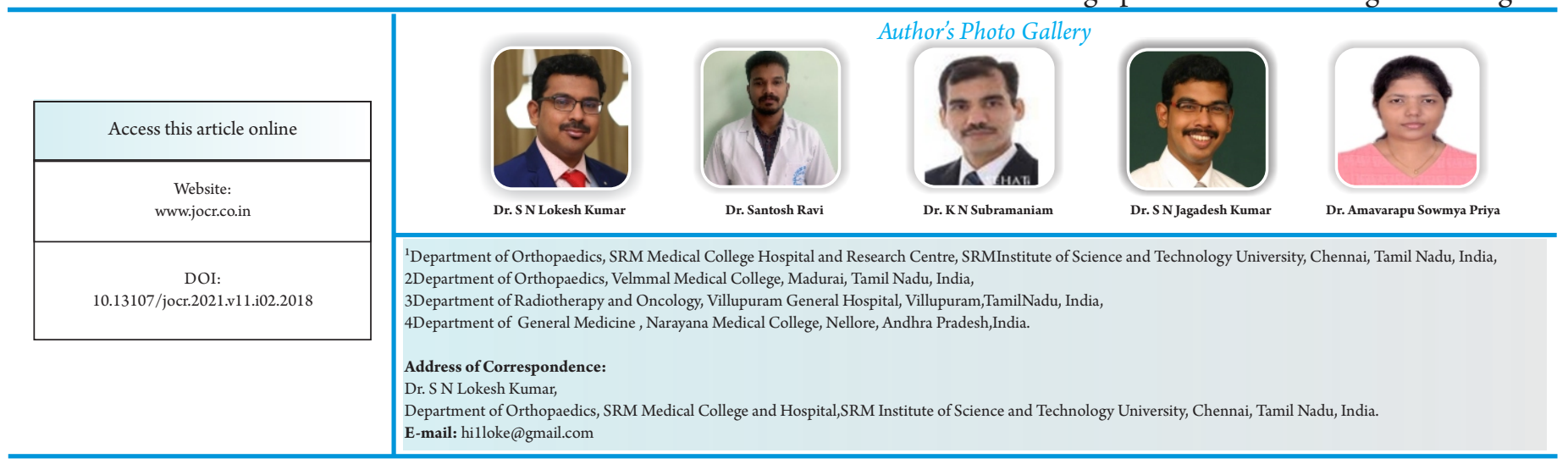

Journal of Orthopaedic Case Reports | pISSN 2250-0685 | eISSN 2321-3817 | Available on www.jocr.co.in | doi:10.13107/jocr.2021.v11.i02.2018 This is an Open Access article distributed under the terms of the Creative Commons Attribution Non-Commercial License (http://creativecommons.org/licenses/by-nc/3.0) which permits unrestricted non-commercial use, distribution, and reproduction in any medium, provided the original work is properly cited. 


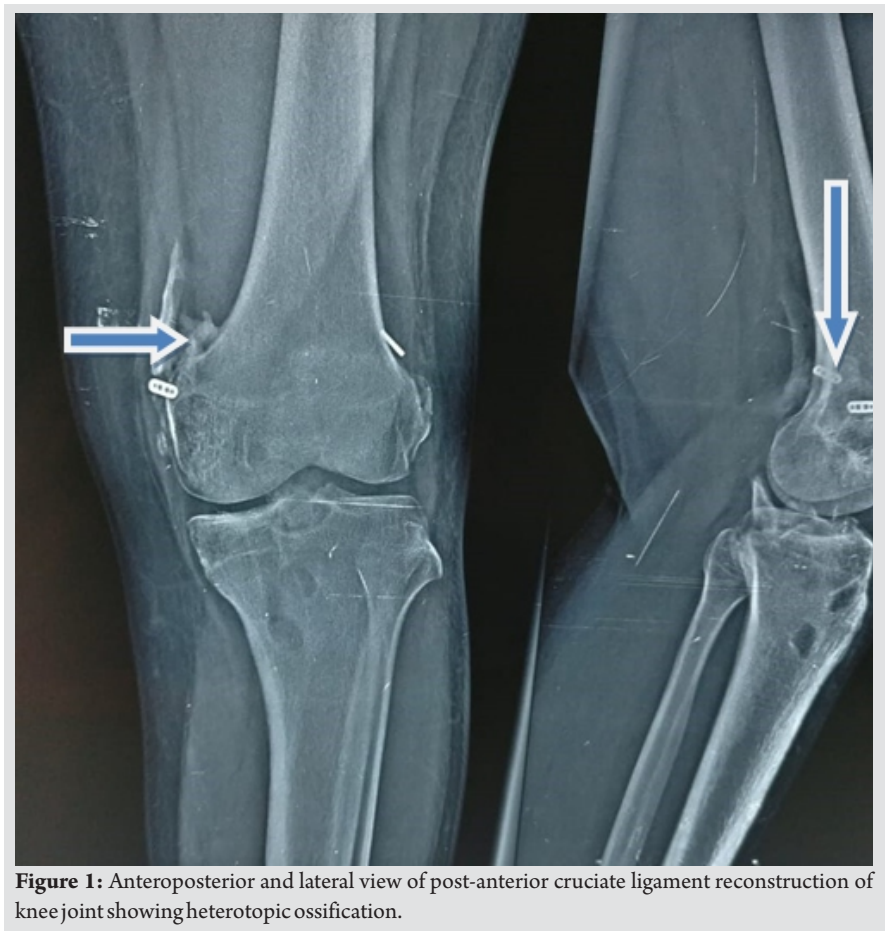

following an in-out technique of femoral canal drilling and a transtibial reconstruction of the PCL with hamstring graft by knee arthroscopy. A partial meniscectomy was performed and ACL graft was fixed with metallic interference screws. After the procedure, knee flexion up to 90 was started immediately on the 1stpost-operative day. Loading was not allowed for 3 weeks. The patient was subjected to our routine rehabilitation program. The patient improved well over 6 months and had full range of movement.

Eight months after the procedure, the patient complained of pain in the lateral side of the knee joint evidencing a slight prominence of hard consistency over the place of the suspensory button. The clinical examination of the knee revealed good stability but on the range of movement in extreme flexion, he developed a clicking sensation over the lateral aspect of the knee joint.

The radiological study showed calcification over the lateral part of the knee joint confirming it to be a case of $\mathrm{HO}$ (Fig.1). The symptoms are mild and the patient was able to manage with most of the activities with no disturbance. Hence, no active intervention was required.

\section{Discussion}

Bone is the only tissue [1] that has an incredible property as such that it can differentiate and develop into its mature form outside its native locations. This is called as HO. In the primary process, there is inappropriate differentiation of pluripotential mesenchymal cells into precursors of osteoblasts [1].

HOis wellrecognized in four major settings mainly trauma, central nervous system damage, burns, and fibrodysplasiaossificans progressiva, a hereditary disorder. Out of these, traumatic events such asfractures, dislocations, and operative procedures around the hip like total hip arthroplasty have proven to increase the formation of $\mathrm{HO}[2]$.

Repeated microtrauma and chronic impingement have been proposed [3] to be the etiology for $\mathrm{HO}$ causing inflammation, hypertrophy, and fibrosis, with the result of a cartilaginous transformation and an ossification. It has also been presumed that these pluripotential stem cells acknowledge local and systemic growth and differentiating osteogenic factors that are released during these microtraumas. Injury is the predisposing risk factor for developing HO. In our case, the patient sustained injury 6 months ago, and hence, we assume that the ACL reconstruction surgery is the causative factor for developing HO. Although the arthroscopic ACL reconstruction is generally viewed as a simple arthroscopic procedure, one has to be aware that even these simple surgical procedures can lead on to $\mathrm{HO}$.

Few studies $[4,5]$ have been seen in the literature describing heterotopic calcifications after a bone-tendon-bone autologous grafting to treat ACL failure. Four cases of heterotopic ossification at the extra-articular exit of the femoral tunnel (with histopathological confirmation) after ACL reconstruction has been reported by Ogilvie-Harriset al. [5] with the bitunnel technique where a metallic screw was used to fix the graft at the femur side. Our case is an illustration of HOfollowing arthroscopic ACL reconstruction where an Endobutton was used to fix the graft at the femoral side and bioabsorbable screws at the tibial side.

There has always been confusion and disagreement between $\mathrm{HO}$ and myositis ossificans. In myositis ossificans, primitive mesenchymal cells differentiate into osteoblasts to form immature bone and it is essentially a reparative process of skeletal muscle. A zonal phenomenon is exhibited by $\mathrm{HO}$ with immature stages of bone formation centrally and more mature trabecular bone peripherally [6].

The ideal protocol for resection of $\mathrm{HO}$ evaluating timing and adjunctive measures such as radiation and indomethacin to prevent recurrence is disputable [7]. A minimum waiting period of 1 year has been agreed by many authors before surgical excision has been carried out [7]. We have monitored the progression of ossification by serial X-ray imaging and our patient didnot show any new symptoms and hence we didnot do any intervention.

Recently, the management options for $\mathrm{HO}$ have been broadly classified as prophylactic or treatment-based strategies. Prophylaxis is aimed to prevent the occurrence or halt the progress of $\mathrm{HO}$, while treatment-based strategies are used for 
improving functional outcome and symptom resolution [8]. Prophylactic radiation is given either $24 \mathrm{~h}$ preoperatively or up to $72 \mathrm{~h}$ postoperatively with a dose range between 400 and $800 \mathrm{cGy}$. A recent study in Germany showed that two-thirds of treating physicians reported customary use of prophylaxis against $\mathrm{HO}$ for high-risk injury patterns [9] but none have used prophylaxis for $\mathrm{HO}$ in cases of arthroscopy.

Studies $[9,10]$ have shown that prophylactic treatment is indicated only in high-risk cases, but this case of $\mathrm{HO}$ in knee arthroscopy might be just an out of the box incident and it may not qualify all arthroscopy surgeries to be high-risk cases for HO. Comparing pre-operative and post-operative radiation dosing in terms of efficacy and complications, no significant difference has been noted so far. However, those who underwent radiation therapy later than $8 \mathrm{~h}$ preoperatively or more than $72 \mathrm{~h}$ postoperatively may show a greater rate of radiographic ossification following surgeries around the hip.

Recent studies $[9,10]$ have shown that there does not appear to be a significant difference between pre-operative and postoperative radiation dosing regarding efficacy or complications, with the exception that those treated more than $8 \mathrm{~h}$ preoperatively or more than $72 \mathrm{~h}$ postoperatively may demonstrate a greater rate of radiographic ossification after a hip surgery.

Recently, concerns over non-union following radiation therapy have cropped up. While nonsteroidal anti-inflammatory drugs (NSAIDs) remain the drug of choice for prophylaxis for $\mathrm{HO}$, prophylactic radiation has its concerns such as joint stiffness and potential oncogenesis. Indomethacin has shown equal efficacy comparable to single-dose radiotherapy in preventing HO after surgeries in the hip in high-risk individuals [11]. However, there are no definitive guidelines regarding the optimal NSAID duration and dosing regimen. In certain cases, corticosteroids are used as a prophylactic modality in fibrodysplasia ossificans progressiva, a hereditary disorder.

Newer treatment modalities [7] that are being evaluated include retinoic acid receptor (RARg) agonists as well as free radical scavengers. Their clinical significance is still being studied. Antiresorptive therapy may increase the risk of developing $\mathrm{HO}$ or may simply delay rather than prevent the bone formation.
Total excision is practically not possible in all cases. Incomplete resection of the HO is associated with recurrence [7]. Softtissue interposition is not of any definitive clinical value. Several authors $[8,9]$ have agreed on various ways to prevent $\mathrm{HO}$ from occurring. These include copious lavage of excessive bone plug reaming following the insertion of patellar bone-tendon-bone ligament constructs. It has also been shown in a prospective study that acquiring adequate hemostasis intraoperatively in the periarticular potential spaces will further minimize either the development and/or progression of $\mathrm{HO}$ following any surgical procedure [10]. The probable reason for $\mathrm{HO}$ in our case is that two ligament reconstructions can potentially cause more hemarthrosis and also much more tissue damage.

Coupens and Yates [11] have shown in their study that the use of post-operative suction drainage significantly reduced the incidence of hemarthrosis, which has also been implicated in contributing to the formation of $\mathrm{HO}$. We recommend all these simple but effective measures could be carried out in the setting of arthroscopic knee surgery to prevent the development of HO. We also like to add that avoiding hand massages to and around the knee joint in the cute setting in non-operative management of knee ligament injuries also reduces the risk of developing HO.

\section{Conclusion}

Numerous studies have suggested ways of preventing and treating this complication. One has to be aware that even these simple surgical procedures can lead on to HO. Minimal invasive surgical procedures like arthroscopy of any joint are not an exception to this unique yet troublesome complication and patient should always be monitored for such problems after surgery.

\section{Clinical Message}

Development of HO in any arthroscopy procedures is a rare phenomenon, yet we have reported such a case. Clinical examination with a mind even for unusual complications will help to prevent such complications at an early stage. HO can also be a cause for poor functional outcomes in less complex surgeries.

\section{References}

1. Berg EE. Tibial bone plug nonunion: A cause of anterior cruciate ligament reconstructive failure. Arthroscopy 1992;8:380-4.

2. Ippolito E, Formisano R, Farsetti P, Caterini R, Penta F. Excision for the treatment of periarticular ossification of the knee in patients who have a traumatic brain injury. $\mathrm{J}$ Bone Joint Surg Am 1999;81:783-9.

3. Ray TD, Lowe WD, Anderson LD, Muller AL, BrogdonBG. Periarticular heterotopic ossification following pharmacologically induced paralysis. Skeletal Radiol 
1995;24:609-12.

4. Patton WC, Tew WM. Periarticular heterotopic ossification after multiple knee ligament reconstructions. A report of three cases. Am J Sports Med 2000;28:398-401.

5. Ogilvie-Harris DJ, Sekyi-Otu A. Periarticular heterotopic ossification: A complication of arthroscopic anterior cruciate ligament reconstruction using a two-incision technique. Arthroscopy 1995; 11:676-9.

6. Binder SM, Rubins IM, Desjardins JV, Zukor DJ, Goltzman D. Evidence for a humoral mechanism for enhanced osteogenesis after head injury. J Bone Joint Surg Am 1990;72:1144-9.

7. Meyers C, Lisiecki J, Miller S, Levin A, Fayad L, Ding C, et al. Heterotopic ossification: A comprehensive review. JBMR
Plus 2019;3:e10172.

8. He SK, Yi M, Zhong G, Cen SQ, Chen JL, Huang FG. Appropriate excision time of heterotopic ossification in elbow caused by trauma. Acta Orthop Traumatol Turc 2018;52:27-31.

9. Armstrong RW, Bolding F, Joseph R. Septic arthritis following arthroscopy: Clinical syndromes and analysis of risk factors. Arthroscopy 1992;8:213-23.

10. Viola RW, Hastings $\mathrm{H} 2$ nd. Treatment of ectopic ossification about the elbow. Clin Orthop Relat Res 2000;370:65-86.

11. Coupens SD, Yates CK. The effect of tourniquet use and hemovac drainage on postoperative hemarthrosis. Arthroscopy 1991;7:278-82.

\section{How to Cite this Article}

Kumar SNL, Ravi S, Subramaniam KN, Kumar SNJ, Priya AS. Heterotopic ossification after knee surgery, arthroscopy is not an exception!!!! Journal of Orthopaedic Case Reports 2021 February; 11(2): 41-44. 